\title{
Recubrimientos bioactivos: Hidroxiapatita y titania
}

\author{
H. MELERO, J. FERNÁNDEZ, J.M. GUILEMANY \\ Centro de Proyección Térmica (CPT) \\ Facultad de Química. Universidad de Barcelona
}

\section{Introducción}

Debido al envejecimiento de la población, como consecuencia del desarrollo de las sociedades occidentales, cada vez son más necesarios dispositivos que permitan que los años de madurez y vejez se vivan de la mejor manera posible. Un ejemplo de estos dispositivos son los empleados en el reemplazo de hueso. Con la edad, el tejido óseo deja de renovarse y las fracturas se multiplican, por lo que son necesarios materiales capaces de cumplir con los requerimientos mecánicos y biológicos a que normalmente es sometido el hueso, sea cual sea su emplazamiento (desde huesos del oído o de la estructura dental a prótesis de hombro o de cadera).

Actualmente, el reemplazo protético más común es el de rodilla, seguido del de cadera y el de hombro. Ya que todas ellas son zonas sometidas a grandes cargas, el primer requerimiento que históricamente se le pidió a una prótesis fue el de la resistencia. Por ello, estas prótesis suelen ser de materiales con unas buenas propiedades mecánicas (en general, metales como aceros inoxidables, aleaciones de cobalto, titanio y aleaciones, etc., que tienen tanto buenas propiedades a traccióncompresión como de tenacidad y ductilidad). Es decir, mientras la prótesis fuera resistente, bastaba con que biológicamente, fuera bioinerte (que no liberara sustancias tóxicas, ni tuviera ningún tipo de interacción con el tejido, creando una cápsula fibrosa alrededor de él). Con el tiempo y la evolución en el campo biotecnológico, se comenzó a buscar que la prótesis tuviera también un valor añadido; es decir, que fuera biocompatible, permitiendo el desarrollo del tejido óseo en contacto con la prótesis, o que, aún más, fuera bioactiva (que indujera la migración y diferenciación de células en células óseas y con ello, que promoviera y es-

Correspondencia:

Hortensia Melero

Centro de Proyección Térmica (CPT)

Universidad de Barcelona, Facultad de Química

C/ Martí i Franqués 1. CP 08028, Barcelona

e-mail: hmelero@cptub.org timulara la creación de nuevo tejido óseo). Para ello, se estudiaron materiales que pudieran tener esta facultad. Como en su mayoría éstos son cerámicos, sin las propiedades mecánicas de las aleaciones metálicas, lo que se consideró fue, salvo en los casos en los que no fueran necesarias tales propiedades mecánicas (huesos del oído medio, relleno de pequeñas cavidades), usarlos para recubrir las prótesis metálicas y mejorar así su osteointegración.

\section{Recubrimientos de hidroxiapatita}

\section{Recubrimientos de hidroxiapatita}

Los fosfatos de calcio constituyen la fase mineral más importante de los tejidos duros de los vertebrados, y desde principios del siglo XX, diferentes estudios han demostrado la similitud entre el mineral óseo y los minerales de fosfatos de calcio con una estructura apatítica. Esto hace que hoy día, estos fosfatos de calcio de origen natural o sintético sean una de las vías más utilizadas en cirugía ósea o dental cuando es necesario un aporte de material (Britel [1]).

Existen, según su fisicoquímica, tres familias de fosfatos de calcio (metafosfatos, pirofosfatos y ortofosfatos), y dentro de esta última familia, diferentes subfamilias según su relación $\mathrm{Ca} / \mathrm{P}$ (TTCP -fosfato tetracálcico-, HAp -hidroxiapatita-, TCP -fosfatos tricálcicos-, OCP -fosfato octacálcico-, etc), pero de todos ellos, el material utilizado en recubrimientos de prótesis, por excelencia, es la hidroxiapatita.

Las apatitas constituyen una familia de compuestos iónicos descrita por la fórmula química M10 (RO4)6 X2 (donde $\mathrm{M}=\mathrm{Ca} 2+, \mathrm{Pb} 2+, \mathrm{Na}+$, etc.; $\mathrm{X}=\mathrm{OH}-, \mathrm{F}-, \mathrm{Cl}-$, etc.; $\mathrm{RO} 4=\mathrm{PO} 43-$, $\mathrm{AsO} 43-$, VO43-, etc.). La X será la que le dé el nombre a la apatita. En los tejidos duros, la apatita presente es una hidroxiapatita; es decir, una apatita donde la $\mathrm{X}$ es un $\mathrm{OH}-$. Así, la fórmula de la hidroxiapatita (de calcio) estequiométrica, de estructura cristalina hexagonal, sería Ca10 (PO4)6 (OH)2.

La hidroxiapatita cálcica se empezó a utilizar clínicamente en los ochenta para mejorar la os- 
teointegración de los implantes, tal y como indica Gallo [2], y desde entonces, han sido extremadamente numerosos los estudios realizados sobre el tema.

Como hoy día se conocen los pros y los contras del uso de estos recubrimientos, es menos común encontrar artículos que estudien recubrimientos puros. Uno de los pocos ejemplos es el de Park et al. [3], que propone el uso de recubrimientos de HAp obtenidos por aerosol - con una salida a gran velocidad - sobre sustratos de $\mathrm{Ti}$, seguidos o no de un tratamiento térmico a $400^{\circ} \mathrm{C}$ durante $1 \mathrm{~h}$, con resultados celulares in vitro que muestran una diferenciación celular inducida respecto al control de $\mathrm{Ti}, \mathrm{y}$ resultados in vivo que promueven la formación de nuevo hueso.

En muchos casos, se está trabajando sobre recubrimientos biomiméticos, más próximos a la estructura apatítica fisiológica y, por tanto, con mejores resultados a nivel biológico. Se suele combinar el procedimiento biomimético (siempre el mismo, introducir el sustrato en una solución supersaturada de iones fosfato y calcio por tiempos varios, a una temperatura de $37^{\circ} \mathrm{C}$ ) con algún tipo de tratamiento. Xie y Luan [4] crearon un nuevo método electroquímico de activación de la superficie, consistente en la inmersión de un sustrato de Ti6Al4V en una solución de $\mathrm{NaOH}$ a $10 \mathrm{M}$ durante $30 \mathrm{~min}$, aplicándole un voltaje de $10 \mathrm{~V}$, seguido de la inmersión en la solución citada, de concentración 5 veces la del plasma sanguíneo, en períodos de 30 minutos, hasta la deposición de alrededor de $1,5 \mu \mathrm{m}$ de espesor de capa, obteniendo capas uniformes de HAp con estructura similar a la del hueso; Yang et al. [5], en cambio, aplican un tratamiento alcalino $\left(48 \mathrm{~h}\right.$ a $60^{\circ} \mathrm{C}$ en una solución de $\mathrm{NaOH}$ a $0,1 \mathrm{M}$ ) y térmico a diferentes tiempos y temperaturas posteriormente a la obtención de la capa de apatita. Utilizan una electrodeposición biomimética, en la que se emplea una solución supersaturada iones calcio y fosfato como electrolito, el sustrato como cátodo y platino como ánodo, durante $60 \mathrm{~min}$, hasta obtener un espesor de capa de unos $20 \mu \mathrm{m}$, obteniendo así capas de apatita con mayor cristalinidad y adherencia al sustrato (hasta 15,6 MPa) que las referencias sin los tratamientos posteriores. Otros recurren a tratamientos térmicos varios. Forsgren et al. [6] realizan un tratamiento térmico previo $\left(1 \mathrm{~h} \mathrm{a} 800^{\circ} \mathrm{C}\right)$, a un sustrato de titanio, para transformarlo a rutilo y aumentar así la adhesión de la siguiente capa de HAp, obtenida por inmersión del sustrato en una solución de PBS ("Phosphate-buffered saline" o solución tampón fosfatada) durante 7 días hasta un espesor de algunos micrómetros, dando lugar a una capa continua de HAp de relativamente alta adherencia al sustrato pero poca al delaminado debido a la alta porosidad (2,4GPa por 'scratch test'). Otra opción es recurrir a una primera capa de material orgánico funcionalizado. Ohtsuki et al. [7] detallan los intentos más importantes que diferentes grupos han realizado al respecto, como promover la formación de grupos silanol $(\mathrm{Si}-\mathrm{OH})$ en el caso de sustratos de vidrios, de grupos Ti-OH, en el caso de sustratos de titanio, siendo mejor si la superficie ha oxidado a anatasa, así como de Ta$\mathrm{OH}$ o $\mathrm{Nb}-\mathrm{OH}$ en los respectivos casos, o grupos carboxilo o sulfónico en el caso de los polímeros; mientras que Takeuchi y colaboradores [8], del mismo grupo, comprueban que la adición de determinadas proteínas de la seda con estructura $\beta$ a una solución de SBF ("simulated body fluid", fluido fisiológico simulado) acelera la formación de un recubrimiento de HAp sobre un polipéptido. También se pueden realizar depósitos sobre sustratos cerámicos utilizados tradicionalmente en determinadas prótesis, como alúmina y circona. Pribosic et al. [9] introducen estas superficies en una solución supersaturada de $\mathrm{Ca} / \mathrm{P}$ durante 27 horas, hasta obtener más de $2 \mu \mathrm{m}$ de espesor de capa, sometiéndolas después a un tratamiento térmico de $1 \mathrm{~h}$ a $1050^{\circ} \mathrm{C}$, lo cual mejora la adherencia al sustrato sin afectar a la bioactividad (como se demuestra al sumergirlas en SBF).

Otra tendencia es trabajar en modificaciones de la HAp. Wood [10] asegura que una superficie con rugosidad nanométrica permite mejor la adhesión de los elementos del medio celular, y por ende de las células; de ahí que pueda favorecer la creación de nuevo tejido. Si esto se aplica a los recubrimientos de hidroxiapatita, la combinación del cerámico bioactivo con la topografía nanométrica es más que prometedora.

Por ello, existen numerosos artículos sobre hidroxiapatita nanoestructurada. Bigi et al. [11], [12] realizaron recubrimientos por vías biomiméticas sobre sustratos de Ti13Nb13Zr y Ti13Nb11Zr, obteniendo capas de 1-2 $\mu \mathrm{m}$ de HAp nanoestructurada tras $3 \mathrm{~h}$ de inmersión en una solución supersaturada de iones calcio y fósforo, que permiten la diferenciación de células mesenquimales en osteoblastos, y una mayor osteointegración respecto al trabajo de Xiong y colaboradores [13], quienes utilizaron procesos hidrotermales con tratamientos térmicos finales a diferentes temperaturas, obteniendo capas de nanopartículas de HAp de espesores de entre 50 y $200 \mathrm{~nm}$. Li et al. [14] emplearon "electron-beam evaporation" para deposi- 
tar capas de HAp nanoestructurada de unos 500 $\mathrm{nm}$ de espesor sobre superficies de Ti anodizado, lo cual dio lugar a una fácil formación de apatita al sumergirlas en $\mathrm{PBS}$ a $37^{\circ} \mathrm{C}$, y a un aumento de la osteointegración in vivo.

En otros estudios, en cambio, se combinan las propiedades osteogénicas de la hidroxiapatita con las de otros compuestos. Una de las vías para ello es la incorporación de iones -como sustitucionesen la estructura de la apatita. Guo et al. [15] depositan una capa de carbonato de calcio sobre un sustrato de Ti6Al4V por electroforesis, que luego se convierte en una apatita hidroxicarbonatada por inmersión en PBS durante lapsos de 1 a 12 días. El resultado de este procedimiento es la obtención de una capa de hidroxiapatita carbonatada con mesoporos, que van desapareciendo con los días de inmersión una vez la conversión es ya completa; así como una gran capacidad de formación de capa de apatita en ensayos de inmersión en SBF.

También se ha multiplicado, en los últimos años, el interés en la comunidad médica por el estroncio. P.J. Marie [16] y P. Ammann [17] aclaran cómo estudios clínicos y farmacológicos concluyen que el ranelato de estroncio estimula la formación de nuevo tejido óseo e inhibe la reabsorción ósea. Así, se han realizado recubrimientos de hidroxiapatita incorporando este elemento en su estructura. Un ejemplo son los obtenidos por Xue et al. [18], que los realizaron con un 10\% molar de iones $\mathrm{Sr}$ (sustituyendo iones $\mathrm{Ca}$ ) por proyección plasma atmosférico sobre sustratos de Ti6Al4V. Los resultados de adherencia son moderados, de 23,5 MPa, y la consistencia de la interfaz recubrimiento-sustrato es buena. Los resultados de inmersión en SBF muestran que la presencia de este elemento resulta favorable para la formación de una capa de apatita sobre la superficie en tiempos cortos (de 1 a 3 días), y también la adhesión, proliferación y diferenciación de osteoblastos en la superficie se mejora respecto a recubrimientos sólo de HAp. Capuccini et al. [19], en cambio, realizan recubrimientos similares (en este caso con porcentajes de sustitución por iones calcio de entre el 0 y el $7 \%$ en producto sólido) por deposición pulsada por láser (PLD) sobre sustratos de Ti, obteniendo resultados muy favorables a nivel celular. A mayores porcentajes de estroncio en el recubrimiento, la actividad de los osteoblastos se estimula y la de los osteoclastos se reduce. Todo ello se suma a las buenas propiedades mecánicas que estudios previos como los de Chen y $\mathrm{Fu}$ [20] han encontrado en la HAp dopada con estroncio -respecto a la no dopada.
Otro elemento interesante es el silicio, componente fundamental de los vidrios bioactivos, que tiene una importancia en la formación de tejido óseo que se ha demostrado ampliamente. Carlisle [21] ya observó que el silicio es un elemento presente en los sitios activos de calcificación en el hueso joven y que, por tanto, está ligado a este proceso. Asimismo, otros estudios como el de Li, Ohtsuki, Kokubo et al. [22] demuestran que se favorece la formación de una capa de apatita por inmersión en SBF sobre la superficie de un gel de sílica. Por ello, son varios los grupos que han intentado introducirla en la estructura de la HAp. Thian et al. [23] han producido recubrimientos de HAp sustituida con iones silicio al 0,8; 2,2 y $4,9 \%$ en peso sobre sustratos de Ti por "magnetron sputtering". La actividad de los osteoblastos sobre las superficies de estos recubrimientos resultó ser superior a la actividad sobre la referencia de HAp. También Xiao et al. [24] han empleado este compuesto en recubrimientos de HAp realizados por proyección plasma al vacío con contenidos de silicio del $0,83 \%$ y $1,26 \%$ en peso. Los resultados de adherencia son moderados, 25,6 MPa, y la interacción de la superficie del recubrimiento con suero humano (HSA, human serum albumin) es mayor que en el caso de recubrimientos sólo de HAp. El mismo grupo realizó también estos recubrimientos por deposición electroforética [25], en este caso con 0,$43 ; 0,81$ y $1,22 \%$ molar de contenido de $\mathrm{Si}$ en sustitución, sometiendo en algunos casos a los recubrimientos a tratamientos térmicos a $800^{\circ} \mathrm{C}$. Aun así, los resultados de adherencia fueron menores que en el caso de proyección plasma, llegando a $16,7 \mathrm{MPa}$ en los mejores casos. De todas maneras, la interacción del recubrimiento con suero bobino (BSA, Bovina serum albumin) fue mejor que en el caso de recubrimientos sólo de HAp.

\section{Recubrimientos de hidroxiapatita con un com- puesto inorgánico}

Otra opción es combinar la HAp con algún compuesto inorgánico. Stojanovic et al. [26] realizan recubrimientos graduales de biovidrio (sistema $\mathrm{SiO} 2-\mathrm{Na} 2 \mathrm{O}-\mathrm{K} 2 \mathrm{O}-\mathrm{CaO}-\mathrm{MgO}-\mathrm{P} 2 \mathrm{O} 5$ ) como matriz y nanopartículas de HAp por electroforesis (a diferentes voltajes y tiempos de deposición) sobre sustratos de Ti6Al4V, lo cual permite una fácil variación de los porcentajes de fases en los recubrimientos obtenidos, y un control de la estructura y la composición de los mismos. También Bharati et al. [27] han estudiado recubrimientos, obtenidos por esmaltado sobre sustratos de Ti6Al4V, con 
biovidrio (sistema $\mathrm{SiO} 2-\mathrm{Na} 2 \mathrm{O}-\mathrm{CaO}-\mathrm{P} 2 \mathrm{O} 5-\mathrm{TiO} 2-$ B2O3) y de un 10 a un $25 \%$ en peso de polvo de HAp (micro- o nanométrico) en la mezcla de partida. Los resultados de adherencias obtenidos por scratch test demuestran que los resultados con polvo de HAp, sobre todo al $10 \%$ en masa para el polvo micrométrico y al $15 \%$ en masa para el nanométrico, son mejores que en recubrimientos sólo con biovidrio, además de observar que la esterilización $\gamma$ influye positivamente en las propiedades mecánicas de estos recubrimientos. Asimismo, Xie et al. [28] estudian recubrimientos de mezclas de un vidrio bioactivo con nanohidroxiapatita en diferentes proporciones, obtenidos por proyección plasma sobre sustratos de Ti6A14V. Los resultados de ensayos in vivo muestran que el contacto hueso-implante y la formación de nuevo hueso es mucho mayor en el caso de implantes recubiertos con estos recubrimientos respecto a implantes no recubiertos, o recubiertos únicamente con HAp, acentuándose la diferencia con el paso de las semanas.

Otros grupos como el de Morks [29] se han centrado en el estudio de la sílica, proyectando mezclas de sílica (al 10 y 20\%) e hidroxiapatita mediante proyección plasma atmosférica de tipo túnel. Esta técnica es una variante de la proyección plasma convencional consistente en un diseño que permite que el plasma se mueva en forma de túnel, de manera que se aumente la eficiencia del recubrimiento. Observaron que la adición de sílica repercutía favorablemente en las propiedades mecánicas del recubrimiento, aumentando la dureza, la resistencia al desgaste -debido a las propiedades autolubricantes de las partículas de sílica-, la densidad y la adherencia del recubrimiento -de 0,6 a $1 \mathrm{MPa}$ en un ensayo de "peel-off". El mismo grupo estudió también mezclas de un $75 \%$ de HAp con un $15 \%$ de sílica y un $10 \%$ de polvo de titanio en peso [30], proyectadas mediante la misma técnica. Posteriormente, se realizaron tratamientos térmicos a 400,600 y $650^{\circ} \mathrm{C}$ en atmósfera de aire. Observaron la influencia del aumento de la intensidad del haz de plasma en la resistencia al desgaste abrasivo y en la dureza, encontrando que al aumentar la densidad del recubrimiento, mejoraban ambas propiedades.

Otros compuestos que también se estudian son los de carbono. Ya en el año 1977 Jenkins y De Carvalho [31] repararon en la excelente biocompatibilidad que presentaba este elemento. Posteriormente, Christel et al. [32] observaron que estos compuestos presentaban unas propiedades mecánicas más cercanas a las del hueso que las de otros materiales. En concreto, uno de los compuestos estudiados son los nanotubos de carbono (CNT): existe cierta controversia, tal y como explican Chen et al. [33], en el uso de estos nanotubos por su posible toxicidad, pero a pesar de ello, según sus defensores, estos recubrimientos aunarían la bioactividad intrínseca de la HAp con la de los CNT, que provendría tanto de su naturaleza como de su estructura nanométrica. Balani et al. [34] han proyectado por plasma atmosférica mezclas de HAp y CNT (4\% en peso) sobre sustratos de Ti6Al4V, con resultados positivos. Las mejoras en las propiedades mecánicas se traducen en una mejora del $56 \%$ en la tenacidad de fractura y del $80,4 \%$ en la cristalinidad; asimismo, el comportamiento biológico es bueno, observando que la presencia de CNT parece favorecer la precipitación de apatita en la superficie del recubrimiento. Por otra parte, las sospechas de toxicidad quedan descartadas por los resultados obtenidos en ensayos celulares, donde se ha visto que el crecimiento y proliferación de osteoblastos se ve promovida. El mismo grupo [35] realizó también más estudios con idénticos recubrimientos, que se centraron en las propiedades tribológicas de los recubrimientos en condiciones fisiológicas. Los resultados del ensayo de pin-on-disk en medio SBF mostraban una mejor resistencia al desgaste de los recubrimientos de HAp y de HAp-CNT respecto al sustrato; y, asimismo, la pérdida de masa y volumen era inferior en el caso de los recubrimientos HAp-CNT respecto a los otros dos.

Kaya [36] realizó recubrimientos similares (HAp con un 0,$5 ; 1$ y $2 \%$ en peso de CNT) por electroforesis sobre sustratos de acero inoxidable, obteniendo mejoras evidentes en las propiedades mecánicas respecto a los recubrimientos sólo de HAp, tanto en dureza, módulo elástico, tenacidad como en resistencia a la cizalla interlaminar. Además, recalcó la conveniencia de realizar tratamientos térmicos, proponiendo uno de $600^{\circ} \mathrm{C}$ durante $2 \mathrm{~h}$ para mejorar la resistencia del recubrimiento a la delaminación.

Otro tipo de recubrimientos que también son interesantes son los compuestos HAp-Ag. Desde hace muchos años se conoce la acción anti-microbiana y anti-bacteriana de la plata, que se ha visto reflejada en estudios como el de Hollinger [37]. Estos compuestos permitirían combinar la bioactividad de la hidroxiapatita con esta acción contra-infección. Chen et al. [38] utilizan el método sol-gel para realizar recubrimientos de HAp con un 1 y un $1,5 \%$ en peso de nitrato de plata sobre sustratos de Ti puro. Los resultados muestran que 
en los recubrimientos con plata, la adhesión de las bacterias S.epidermidis y S.aureus es mucho menor que en recubrimientos de HAp, mientras que los resultados de ensayos de diferenciación celular con osteoblastos muestran que no existen demasiadas diferencias entre el recubrimiento de HAp y el que contiene un $1 \%$ de nitrato de plata, siendo ambos osteoconductivos. Así, los recubrimientos mixtos aunarían ambas propiedades. Noda et al. [39], en cambio, realizan recubrimientos de HAp con un $3 \%$ en peso de óxido de plata mediante proyección térmica atmosférica por llama sobre sustratos de Ti puro. Los resultados muestran una elevada acción antibacteriana (la bacteria empleada fue un tipo de S.aureus, el MRSA B++); así como una presumible osteoconductividad debido a la capacidad que mostraron estos recubrimientos para formar una capa de apatita sumergidos en SBF; y una importante liberación de iones plata tras $24 \mathrm{~h}$ de implantación.

\section{Recubrimientos de hidroxiapatita con compuestos orgánicos}

Tal y como citan Teng et al. [40], en los últimos años se ha considerado la posibilidad de combinar HAp con algún tipo de polímero bioabsorbible. En concreto, proponen que este polímero sea el colágeno, ya que el colágeno tipo I es la molécula estructural más importante de los tejidos duros. Kim et al. [41], por ejemplo, recalcan su papel esencial en la respuesta celular, y Rammelt et al. [42] realizan estudios sobre su influencia en la remodelación ósea. Por ello, Teng realiza capas nanocompuestas de colágeno y unas proporciones de 10, 20 ó 30\% de HAp en peso por formación del sol en condiciones biomiméticas y aplicación sobre sustratos de Ti por "spin-coating". Al aumentar el porcentaje de HAp, el ángulo de contacto es menor (la hidrofilicidad mayor) y la proliferación y diferenciación de los osteoblastos mayor. Manara et al. [43] estudian recubrimientos similares obtenidos mediante deposición electroquímica a diferentes tiempos (de $5 \mathrm{~s}$ a $30 \mathrm{~min}$ ), obteniendo estructuras interesantes como la de nanocristales apatíticos rodeando fibras de colágeno, todas ellas con elevada afinidad con la fibronectina. También grupos como el de Daugaard et al. [44] han comparado recubrimientos obtenidos por deposición electroquímica en condiciones fisiológicas de una capa interna de HAp con una capa externa de fibras de colágeno frente a recubrimientos sólo de HAp obtenidos por electrodeposición y por proyección térmica plasma sobre sustratos de Ti6Al4V en forma de cilindro, que se han inser- tado en un húmero de perro. Tras cuatro semanas de observación, se ve que la presencia de HAp ha resultado en una mejora de la fijación mecánica. Los mejores resultados de la resistencia a la cizalla y del crecimiento de hueso se dan con los dos recubrimientos de HAp; sin embargo, no se dan mejores resultados con los recubrimientos con colágeno, contrariamente a estudios anteriores como el citado de Rammelt u otros del mismo autor [45], donde se detalla también el aumento del proceso de remodelación ósea en las zonas de un implante recubiertas de colágeno. Como se ha constatado, las débiles propiedades mecánicas de estos recubrimientos hace necesario realizar mayor número de estudios, como el de Kikuchi et al. [46], centrados en mejorar las propiedades mecánicas de nanocompuestos de HAp y colágeno mediante su reticulación con glutaraldehído.

Otros grupos que han intentado idear recubrimientos compuestos que pudieran optimizar su función, como el de Sun et al. [47], proponen la deposición por electroforesis de recubrimientos graduales sobre sustratos de grafito. En concreto, la de capas de quitosano intercaladas con capas de una matriz de quitosano con nanopartículas de HAp, en un porcentaje al peso que va del 40 al $80 \%$, con la posibilidad de añadir una capa suplementaria de quitosano-heparina. Los resultados dan fe de la eficiencia en la deposición del recubrimiento, y de la posibilidad del control de los espesores del mismo. Estos recubrimientos podrían constituir una nueva vía de investigación, teniendo en cuenta otros estudios como el de Lin et al. [48], que recalcan la hemocompatibilidad del complejo quitosano-heparina.

Otra propuesta es la de Negroiu et al. [49], que estudian recubrimientos de un compuesto de HAp y un copolímero maleato de sodio sobre sustratos de Ti, mediante la técnica de evaporación láser (MAPLE, Matrix Assisted Pulsed Laser Evaporation). Los resultados in vitro demuestran que la viabilidad y proliferación celular se ve promovida en estos recubrimientos respecto a los de HAp o a los propios sustratos.

Se están estudiando también los bifosfonatos en combinación con la HAp. Fleisch y su grupo [50], [51] ya habían demostrado la capacidad de los bifosfonatos para inhibir la reabsorción ósea mediante su acción, no aclarada, sobre los osteoclastos. Oliveira et al. [52] realizan recubrimientos de apatita, obtenidos biomiméticamente por inmersión en una solución de SBF y de $1,5 \mathrm{SBF}$ durante 14 días, impregnados de un bifosfonato, el clodronato de sodio, mediante la adición 
del bifosfonato a diferentes concentraciones sobre el recubrimiento, y tres horas después, un secado a $60^{\circ} \mathrm{C}$. Los resultados muestran que la presencia de este bifosfonato a determinadas concentraciones -las dos menores- estimula la viabilidad celular respecto a la referencia. El efecto positivo de los bifosfonatos en la osteointegración de un implante se ve refrendado también por estudios como el de Jakobsen et al. [53], donde se constata que la inyección local de un bifosfonato, en el sitio de implantación de una prótesis de Ti6Al4V con un recubrimiento de HAp obtenido por proyección plasma, lleva a un aumento del volumen del hueso en la zona del peri-implante y de la resistencia a la cizalla, aunque no se observen grandes diferencias en la zona de contacto hueso-implante.

Mención aparte merecen las mezclas realizadas por Sanpo y Khor [54]. Este grupo propone el empleo de mezclas de un polvo nanométrico de HAp dopada con Ag y un polvo de PEEK, para proyectarlas mediante proyección fría sobre un sustrato de vidrio. Los resultados muestran una capacidad antibacteriana, mayor a mayor porcentaje de HAp-Ag en la mezcla, que no ha variado de antes a después de la proyección.

\section{Recubrimientos de óxido de titanio}

\section{Recubrimientos de óxido de titanio}

Tradicionalmente, el uso que se le ha dado al óxido de titanio es distinto al biomédico, explotándose principalmente sus propiedades fotocatalíticas (Linsebigler et al. [55]), mientras que sus propiedades biológicas han sido mucho menos analizadas. A priori, es un compuesto no bioactivo. Algunos estudios lo consideran bioinerte, mientras que otros defienden que permite la formación de hueso sobre su superficie sin formar una cápsula fibrosa (biocompatible), aunque no la favorezca (no osteoinductivo, y por ende no bioactivo). Todos estos estudios combinan el TiO2 con otro compuesto, o le aplican algún tratamiento o condiciones especiales para aprovechar sus otras propiedades (mecánicas, anticorrosión...) en usos biomédicos.

Se puede decir que, de por sí, todas las prótesis de titanio tienen una capa de óxido de titanio sobre su superficie, ya que se forma de manera natural, $\mathrm{y}$ es ella la que interacciona con el medio, siendo bioinerte, tal y como afirman Breme [56] y Ochsenbein [57]. Los estudios de Jaworski et al. [58] han concluido que los recubrimientos de $\mathrm{TiO} 2$ tienen unas buenas propiedades mecánicas. Asimismo, a raíz de los estudios de Lamaka et al. [59], que proponen el uso de recubrimientos de $\mathrm{TiO} 2$ sobre sustratos de $\mathrm{Al}$ por su elevada resistencia a la corrosión, Chiu et al. [60] realizaron recubrimientos de anatasa sobre sustratos de NiTi por sol-gel, seguido de dip-coating y de un tratamiento hidrotermal $\left(105^{\circ} \mathrm{C}\right.$ durante $\left.24 \mathrm{~h}\right)$, con excelente resistencia a la corrosión. Tomando como base estos resultados, Shi et al. [61] muestran cómo recubrimientos de $\mathrm{TiO} 2$ depositados por dip-coating, en una solución precursora del $\mathrm{TiO} 2$ a $50^{\circ} \mathrm{C}$ y con un tratamiento hidrotermal a $120^{\circ} \mathrm{C}$, durante un período entre 7 y 24 horas, sobre sustratos de magnesio oxidados por oxidación mediante micro-arco (MAO, micro-arc oxidation), tienen una resistencia a la corrosión en medio biológico (solución Hank's) muy superior a la del sustrato sin $\mathrm{TiO} 2$.

Pero desde el punto de vista biológico, es conveniente señalar que, de acuerdo con Uchida et al. [62], se sospecha que la nucleación de una capa de apatita sobre el óxido de titanio depende mucho de la orientación cristalográfica de los planos de la apatita y del óxido de titanio, y se favorecería más en la anatasa que en el rutilo. Algunos estudios, para mejorar el comportamiento biológico, recurren simplemente a tratamientos físicos o químicos de un recubrimiento ya realizado de óxido de titanio, en cualquiera de sus fases. Por ejemplo, se le puede realizar un tratamiento alcalino al recubrimiento de $\mathrm{TiO} 2$, como proponen Chen et al. [63], que muestran la capacidad de formar una capa de apatita en su superficie tras inmersión en SBF durante 14 a 28 días, contrariamente a los recubrimientos no tratados. Li et al. [64] atribuyen este hecho a la multiplicación de los grupos funcionales Ti-OH sobre la superficie. Este aumento de la bioactividad con estos tratamientos se observa también en otros artículos, como el de Wei et al. [65]. Otra posibilidad es irradiar los recubrimientos de $\mathrm{TiO} 2$ con luz ultravioleta. Esta luz tiene la capacidad de inducir la creación sobre la superficie de abundantes grupos Ti-OH, tal y como explican Henderson [66] o Liu et al. [67], lo cual favorece la formación de una capa apatítica. Asimismo, estudios histológicos realizados por los segundos muestran que tras $168 \mathrm{~h}$ de irradiación UV, los recubrimientos permiten, no sólo la adhesión de hueso al recubrimiento, sino la formación de nuevo hueso tras 2 meses de implantación, contrariamente a los recubrimientos no irradiados.

Otros grupos intentan otras vías para aunar bioactividad y adhesión en estos recubrimientos. Brohede et al. [68] depositan un recubrimiento gradual por PVD sobre sustratos de Ti, variando el caudal de oxígeno, de manera que se obtenga ana- 
tasa en la superficie y titanio en la interfaz de contacto con el sustrato, con variación gradual en la composición de uno a otro extremo. Se consigue un valor de adherencia de 1,69 $\mathrm{GPa}$, muy superior al requerido según normas. Además, las pruebas de inmersión en SBF mostraron la formación de capa de apatita en superficie tras la primera semana.

Sin duda, el modo más prometedor de obtener una bioactividad con recubrimientos de $\mathrm{TiO} 2$ es realizarlos de manera que tengan una estructura nanométrica. Recientemente se ha reconocido, a partir de los trabajos de Cavalcanti-Adam et al. [69] o Von der Mark et al. [70], la importancia de la organización nanométrica de las señales extracelulares de la matriz en la respuesta celular: Las distancias entre receptores celulares para un determinado péptido y la organización espacial de éstos están a escala celular, y esto ha hecho que la mayoría de aproximaciones actuales a recubrimientos de óxido de titanio sean a escala nanométrica. Ochbensein et al. [57] consiguen recubrimientos de óxido de titanio (rutilo/anatasa) nanoestructurados sobre sustratos de Ti, mediante la técnica sol-gel, con aplicación del sol por spincoating seguido de un recocido a $450^{\circ} \mathrm{C}$ durante $1 \mathrm{~h}$. Los resultados de ensayos in vitro realizados con células constatan el buen comportamiento de las mismas sobre estos recubrimientos, su buena adhesión y proliferación. Advincula et al. [71] han estudiado la formación de recubrimientos de $\mathrm{TiO} 2$ nanoestructurado por sol-gel sobre sustratos de Ti6A14V, activando previamente la superficie mediante la inmersión durante 10 minutos en solución "piraña", que promueve la formación de radicales $\mathrm{OH}-$ en la superficie. Los resultados de adherencia son extraordinariamente elevados, 76 $\mathrm{MPa}$; y se combinan con una buena resistencia a la corrosión y la pronta formación de una capa de apatita en superficie, con precipitación ya tras el primer día; así como con la capacidad de ejercer de barrera eficaz contra la liberación de iones metálicos del sustrato.

Sin embargo, la vía más prolífica, actualmente, en recubrimientos nanoestructurados de óxido de titanio es el empleo de nanotubos de $\mathrm{TiO} 2$ obtenidos por oxidación anódica sobre sustratos de Ti, que se hacen crecer directamente sobre la superficie de titanio, de manera que se cree una unión directa -química- entre los nanotubos de óxido y el titanio, y de ahí que se obtengan buenas adherencias, tal y como aclaran Crawford et al. [72]. El mismo autor menciona $[72,73,74]$ asimismo que la principal ventaja de estos nanotubos son sus propiedades mecánicas, de gran parecido con las del hueso. Von Wilmowski et al. [75] han obtenido recubrimientos como los citados para hacer estudios biológicos. Ensayos in vivo en cerdos a diversos tiempos entre 3 y 90 días tienen resultados que muestran, en primer lugar, pocas diferencias en la superficie de contacto hueso-recubrimiento y en la segregación de osteocalcina entre las muestras recubiertas con nanotubos y las de Ti. Sí se observa, en cambio, mayor segregación de colágeno tipo I en las muestras con nanotubos, lo cual indica una promoción de la diferenciación celular. Ma et al. [76] realizan recubrimientos de nanotubos de $\mathrm{TiO} 2$ sobre Ti, sometiéndolos después a un tratamiento térmico $\left(2 \mathrm{~h} \mathrm{a} 500^{\circ} \mathrm{C}\right)$ de cristalización y a una pre-calcificación por inmersión en una solución de Na2HPO4 a $0,5 \mathrm{M}$ durante $24 \mathrm{~h}$ y en una de $\mathrm{Ca}(\mathrm{OH}) 2$ saturada durante $5 \mathrm{~h}$. Los resultados muestran la presencia de una capa de apatita en superficie tras los primeros 7 días, contrariamente a los nanotubos sin precalcificar, los cuales presentan resultados mucho más pobres.

\section{Recubrimientos de óxido de titanio con otros com- puestos}

Huang et al. [77] proponen el uso de recubrimientos de $\mathrm{TiO} 2$ obtenidos mediante deposición atómica sobre nanofibras de Si depositadas sobre sustratos de Ti. Los resultados in vitro muestran una mejora de la proliferación celular respecto a las referencias, además de un aumento de la diferenciación celular y la mineralización.

Bienias et al. [78], Ochbensein [57], Aäritalo [79] y Areva et al. [80] estudian intercapas de $\mathrm{SiO} 2-\mathrm{TiO} 2$ sobre sustratos de Ti y Ti6Al4V, observando que la intercapa mixta produce una mejora de la adherencia del recubrimiento, que la proliferación celular (fibroblastos y osteoblastos) se ve favorecida por estos recubrimientos y que hay, también, una prolongada actividad osteoblástica que lleva a una formación rápida de matriz extracelular y, por tanto, de hueso. Los resultados de inmersión en SBF muestran una aparición de apatita en superficie, aunque lenta.

Wei et al. [81] realizan recubrimientos compuestos de óxido de titanio con $\mathrm{CaTiO} 3$ sobre sustratos de Ti6Al4V por oxidación plasma electroquímico (PEO, plasma electrochemical oxidation), con post-tratamientos en medio alcalino $\left(24 \mathrm{~h} \mathrm{a} 60^{\circ} \mathrm{C}\right.$ en una solución a $\left.5 \mathrm{M}\right)$ y a alta temperatura $\left(1 \mathrm{~h} \mathrm{a} 800^{\circ} \mathrm{C}\right)$, que en los primeros siete días de inmersión en SBF permiten la precipitación en superficie de una capa de apatita.

Como en el caso de la HAp, otro compuesto que se añade, en ocasiones, a los recubrimientos 
de óxido de titanio es la plata (ver Necula et al. [82]), por sus propiedades antimicrobianas y antibacterianas (ver lo dicho por Hollinger más arriba [37]), En este caso la Ag se encuentra en forma de nanopartículas infiltradas en la capa de óxido (anatasa/rutilo) obtenida por oxidación electrolítica por plasma. Los resultados muestran la eliminación total de bacterias como el Staphylococcus aureus durante las primeras 24 horas de implantación.

Yuan et al. [83] han hecho algún intento de realizar recubrimientos de óxido de titanio con butóxido de titanio sobre acero inoxidable vía solgel, obteniendo muy buena resistencia a la biocorrosión y capacidad de formar una capa apatítica en su superficie.

\section{Recubrimientos de HA/TiO2}

\section{En capas separadas}

Zhang et al. [84], Wen et al. [85] y He et al. [86] realizan recubrimientos vía sol-gel aplicados mediante dip-coating o spin-coating, respectivamente, que consisten en una capa de $\mathrm{TiO} 2$ sobre el correspondiente sustrato seguida de una capa de HAp, encontrando que tras el primer día se induce la formación de una capa de apatita sobre la superficie.

Otra posibilidad es la que emplean Tomaszek et al. [87]; la de obtener recubrimientos multicapa vía proyección térmica, por proyección plasma de suspensión (suspension plasma spraying) sobre sustratos de Ti, con cierto control de la aparición de fases derivadas de la hidroxiapatita no deseadas, según la potencia aplicada.

Lee et al. [88] realizan recubrimientos similares mediante la deposición por haz de electrones (electron-beam deposition) a escala nanométrica sobre sustratos de Ti, sometiendo el recubrimiento de $\mathrm{TiO} 2$ a $500^{\circ} \mathrm{C}$ durante $2 \mathrm{~h}$ y el conjunto de los dos a temperaturas entre 450 y $600^{\circ} \mathrm{C}$ durante $2 \mathrm{~h}$. Los resultados muestran una mejora en la adherencia del recubrimiento para los diferentes tratamientos térmicos respecto a los recubrimientos de HAp, manteniéndose la adherencia tras un día de inmersión en SBF. La velocidad de disolución es menor que en el caso de la HAp sola, y el comportamiento celular in vitro es similar al obtenido con recubrimientos de HAp, de lo cual se deduce que hay buena proliferación y diferenciación.

Wang et al. [89] consideran la aplicación de una capa de nanotubos de $\mathrm{TiO} 2$ (anatasa) por anodizado sobre Ti seguida de un tratamiento térmico a $500{ }^{\circ} \mathrm{C}$, y tras ella, la obtención de HAp por de- posición electroquímica seguida de la inmersión en $\mathrm{NaOH}$ a $0,1 \mathrm{~mol} / \mathrm{L}$ durante $2 \mathrm{~h}$. Se mejora, con ello, la adherencia del recubrimiento respecto a los recubrimientos sólo de HAp por el entrelazado de los cristales de HAp entre los nanotubos de TiO2.

Albayrak [et al.] [90] emplean la deposición electroforética para obtener estos recubrimientos sobre Ti6A14V: se deposita la capa de TiO2 (anatasa) seguida de la de HAp (ambas con estructura nanométrica), y se aplica finalmente un tratamiento térmico de sinterización a $1000^{\circ} \mathrm{C}$. Así se obtienen mejoras en la resistencia a la cizalla en los recubrimientos mixtos a menores voltajes y se evita la descomposición de la HAp a altas temperaturas.

Intentos de grupos como el de Zhu et al. [91] de conseguir recubrimientos tri-capa sobre sustratos de $\mathrm{Ti}$, con una capa interna de $\mathrm{TiO} 2$ densa (anatasa) formada por oxidación preanódica; una capa media formada por $\mathrm{TiO} 2$ porosa (rutilo/anatasa) formada por MAO ("microarc oxidation"); y una capa externa formada por HAp obtenida por vía hidrotermal, han desembocado en excelente resistencia a la corrosión. Además, estos recubrimientos presentan una excelente capacidad de adsorción para las proteínas, y buenos resultados tras ensayos celulares in vitro (buena proliferación y diferenciación celular -resultados tras 28 días) e in vivo (capacidad de formación de tejido óseo tras 30 días).

Sun y Wang [92] realizan estos recubrimientos multicapa de forma biomimética sobre sustratos de Ti o de NiTi: forman una primera capa de $\mathrm{TiO} 2$ (anatasa) por oxidación con $\mathrm{H} 2 \mathrm{O} 2$ y técnicas de envejecimiento ("hot water aging technique"), y después inducen la formación de una capa de apatita por un proceso biomimético acelerado (inmersión en una solución $5 \mathrm{SBF}$ por varios períodos de tiempo). El resultado es una buena interfaz recubrimiento-sustrato.

\section{En la misma capa}

Se han hecho estudios sobre la idoneidad de crear recubrimientos que contengan hidroxiapatita y titania mezclados.

Los artículos que se inclinan por hacerlo vía sol-gel obtienen resultados esperanzadores. Im et al. [93] obtienen recubrimientos de HAp y TiO2 (a $10,30,50$ y $70 \%$ molar) vía sol-gel aplicados mediante spin-coating a $2000 \mathrm{rpm}$ durante $10 \mathrm{~s} \mathrm{y}$ sometidos a un tratamiento térmico a $500^{\circ} \mathrm{C}$ durante $2 \mathrm{~h}$, sobre sustratos de Ti. Los resultados muestran tener unas buenas adherencias al sustrato (evaluadas por "scratch test"), mejores cuanto mayor porcentaje de $\mathrm{TiO} 2$ se emplea; asimismo, 
se comprueba la capacidad de formación de una capa apatítica (aunque para tiempos posteriores a una semana), y se ve que el comportamiento celular (la adhesión cuanto menos) es buena y también mejora con el aumento del porcentaje de $\mathrm{TiO} 2$.

Han, Yu y Zhou [94] realizan otros intentos sobre sustratos de Ti, vía sol-gel con aplicación por spin-coating a $1500 \mathrm{rpm}$ durante $20 \mathrm{~s}$, con proporciones molares de $80 \% \mathrm{HA} / 20 \% \mathrm{TiO} 2$, seguido de tratamientos térmicos a varias temperaturas entre 450 y $1200{ }^{\circ} \mathrm{C}$. Los resultados demuestran que el efecto del óxido de titanio es un aumento de la consistencia y el ajuste en la interfaz recubrimiento-sustrato, de la adherencia del recubrimiento (con tratamientos térmicos a $1200^{\circ} \mathrm{C}$ se llegan a valores de $50 \mathrm{MPa}$ ), además de un aumento en la temperatura de cristalización de la HAp, y una mejora de la resistencia a la corrosión. También se observa que el comportamiento en condiciones fisiológicas es bueno: el recubrimiento tiene capacidad formadora de capa de apatita tras 2 días de inmersión, y los resultados in vitro muestran una buena proliferación osteoblástica. También Nathanael et al. [95] realizan recubrimientos compuestos de $\mathrm{HAp} / \mathrm{TiO} 2$ nanoestructurado a diferentes proporciones $(0,10,20,50,80$ y $100 \%$ de $\mathrm{TiO} 2$ en volumen) sobre sustratos de vidrio, mediante la técnica sol-gel, aplicándolo por dip-coating seguido de un tratamiento térmico a $500^{\circ} \mathrm{C}$ durante 1h. Los resultados muestran recubrimientos más uniformes, menos rugosos, y con mejores propiedades mecánicas (módulo elástico, dureza y adherencia) a mayores porcentajes de $\mathrm{TiO} 2$, ya que esta fase ejerce de matriz sobre la cual las nanopartículas de HAp se pueden depositar más eficientemente y más homogéneamente.

También hay grupos como el de Gaona, Lima y Marple [96], que proyectan recubrimientos mixtos por proyección térmica sobre sustratos de Ti6Al4V; en concreto, por HVOF con propileno. Se trata de recubrimientos nanoestructurados, de $\mathrm{TiO} 2$, al 80 o $90 \%$ en peso, en forma mayoritariamente de rutilo tras la proyección, con HAp. Las propiedades mecánicas del recubrimiento, como la adherencia (valores de $68 \mathrm{MPa}$ con un $20 \%$ de HAp, y valores superiores a la resistencia del pegamento con un $10 \%$ de HAp) o la dureza mejoran respecto a los recubrimientos de HAp, y son mejores a menor porcentaje de la misma. Resultados in vitro de recubrimientos similares (con el 10\% de HAp en peso) obtenidos por el mismo grupo [97] muestran un comportamiento celular (adhesión, proliferación y diferenciación celular) muy bueno, mejor que en el caso de recubrimientos de HAp obtenidos por plasma o de sustratos de Ti6Al4V sin recubrir.

Otra manera se obtener estos recubrimientos es por "sputtering": determinados grupos, como el de Boyd et al. $[98,99]$ depositan por "sputtering" recubrimientos de titanio seguidos de recubrimientos de fosfatos de calcio sobre sustratos de Ti-6Al-4V. Mediante la aplicación de tratamientos térmicos, se provoca la difusión del óxido de titanio formado a través del recubrimiento poroso de fosfato cálcico, lo cual da lugar a recubrimientos de composiciones graduales con muy buen comportamiento celular en su superficie.

Kim et al. [100] han empleado la MAO (microarc oxidation) combinada con deposición electroforética: por MAO, se forma un recubrimiento poroso de $\mathrm{TiO} 2$ sobre una superficie de Ti mientras por EPD partículas de HAp cargadas negativamente migran a través de los poros del $\mathrm{TiO} 2$. Las superficies obtenidas de esta manera muestran una importante diferenciación celular.

\section{Otras combinaciones}

Existen otros intentos de combinar hidroxiapatita sustituida con iones flúor con $\mathrm{TiO} 2$. Estudios previos como el de Cheng et al. [101] detectaron que el F- mejora la estabilidad de la HAp, así como su bioactividad. Promueve la mineralización en el proceso de formación de nuevo tejido óseo, tal y como demuestran estudios como el de Kim et al. [102], así que intentos como el de Wang et al. [103] de depositar estos recubrimientos mixtos sobre Ti vía electrodeposición seguida de una inmersión en $\mathrm{NaOH}$ a $0,1 \mathrm{M}$ a $60^{\circ} \mathrm{C}$ durante $48 \mathrm{~h}$ y de una calcinación en vacío a $650^{\circ} \mathrm{C}$ durante $2 \mathrm{~h}$ demuestran que la adición de iones F- y de TiO2 mejora la cristalinidad de la HAp y disminuye el tamaño de cristal; asimismo, la adherencia del recubrimiento mejora y el ratio de disolución disminuye, mientras que la bioactividad in vitro es similar a la de recubrimientos de HAp.

También existen grupos como el de Mo et al. [104] que realizan, sobre Ti, recubrimientos nanoestructurados de HAp con $\mathrm{Ag}+$ combinada con $\mathrm{TiO} 2$ (anatasa) por inmersión, seguida de un secado a $150^{\circ} \mathrm{C}$ y de un tratamiento térmico a $400^{\circ} \mathrm{C}$ $750^{\circ} \mathrm{C}$ en vacío durante $10 \mathrm{~min}$ : los efectos antibacterianos del recubrimiento son patentes al eliminar tras $24 \mathrm{~h}$ más del $99 \%$ de las bacterias estudiadas.

\section{Conclusión}

Existen muchas otras combinaciones de materiales que pueden ser útiles o recomendables para 
realizar recubrimientos bioactivos, pero requerirían otro estado del arte. En cuanto a las técnicas empleadas, se puede decir, teniendo en cuenta sus características y los resultados observados, que hoy día la vía más eficaz y rápida para obtener recubrimientos de biocerámicos de ciertos espesores es la proyección térmica: a pesar de las ventajas que pueden presentar los otros métodos (en particular, las vías biomiméticas, las más convenientes para obtener recubrimientos con excelente biocompatibilidad por su similitud con el tejido óseo, pero poco viables industrialmente por la lentitud con la que se consiguen capas de cierto espesor), el único que permite realizar estos recubrimientos de forma automatizada, rápida y eficiente es la proyección térmica en todas sus variantes, $\mathrm{y}$, según el caso, determinados procesos de conversión como el anodizado o la MAO. El rango de composiciones utilizable es limitado (notable en el caso de las biomoléculas), pero para poder utilizar industrialmente otros métodos sería necesario optimizar los equipamientos de tal manera que la producción compensara el tiempo y el dinero empleado.

Una vez esto definido, se debería pensar en optimizar siempre los parámetros y las composiciones empleados en estas técnicas, para mejorar en lo posible los resultados clínicos.

Para ello, la primera medida sería no optar por la simple proyección plasma, ya que presenta, como ya se ha dicho, muchos inconvenientes; sino por HVOF o CGS optimizadas.

\section{Referencias}

1. Britel, O. Modélisation et optimisation par la méthodologie des plans d'expérience de la synthèse de l'hydroxyapatite phosphocalcique, du phosphate tricalcique apatitique et du phosphate de calcium apatitique carbonate. Thèse doctorale, 2007

2. Gallo, J. [et al.] Poor survival of ABG I hip prosthesis in younger patients. Biomedical Papers of the medical faculty of the University Palacky, vol. 152, issue 1, p. 163-168 (2008)

3. Park, D.S. [et al.] Improved biocompatibility of hydroxyapatite thin films prepared by aerosol deposition. Journal of Biomedical Materials Research. Part B: Applied biomaterials, vol. 94B, issue 2, p. 353-358 (2010)

4. Xie, J.; Luan, B.L. Formation of hydroxyapatite coating using novel chemo-biomimetic method. Journal of Materials Science: Materials in Medicine, vol.19, issue 10, p. 3211-3220 (2008)

5. Yang, $\mathbf{X}$. [et al.] Biomimetic Ca-P coating on precalcified Ti plates by electrodeposition method.
Applied surface science, vol. 256, issue 9, p. 2700-2704 (2010)

6. Forsgren, J. [et al.] Formation and adhesion of biomimetic hydroxyapatite deposited on titanium substrates. Acta Biomaterialia, vol. 3, issue 6, p. 980-984 (2007)

7. Ohtsuki, C.; Kamitakahara, M.; Miyazaki, T. Coating bone-like apatite onto organic substrates using solutions mimicking body fluid. Journal of Tissue Engineering and Regenerative Medicine, vol. 1, issue 1, p. 33-38 (2007)

8. Takeuchi, A. [et al.] Biomimetic deposition of hydroxyapatite on a synthetic polypeptide with $\beta$ sheet structure in a solution mimicking body fluid. Journal of Materials Science: Materials in Medicine, vol.19, issue 1, p. 387-393 (2008)

9. Pribosic, I.; Klopcic, S.B.; Kosmac, T. Biomimetic preparation and characterization of bioactive coatings on alumina and zirconia ceramics. Journal of the American Ceramic Society, vol. 93, issue 1, p. 288-294 (2010)

10. Wood, M.A. Colloidal lithography and current fabrication techniques producing in-plane nanotopography for biological applications. Journal of the Royal Society Interface, vol. 4, issue 12, p. 1-17 (2007)

11. Bigi, A. [et al.] In vitro culture of mesenchymal sells onto nanocrystalline hydroxyapatite-coated Ti13Nb13Zr alloy. Journal of Biomedical Materials Research Part A, vol. 82A, issue 1, p. 213221 (2007)

12. Bigi, A. [et al.] The response of bone to nanocrystalline hydroxyapatite-coated Ti13Nb11Zr alloy in an animal model. Biomaterials, vol.29, issue 11, p. 1730-1736 (2008)

13. Xiong, J. [et al.] Nanohydroxyapatite coating on a titanium-niobium alloy by a hydrothermal process. Acta Biomaterialia, vol.6, issue 4, p.15841590 (2010)

14. Li, Y. [et al.] The biocompatibility of nanostructured calcium phosphate coated on micro-arc oxidized titanium. Biomaterials, vol.29, issue 13, p.2025-2032 (2008)

15. Guo, Y.; Zhou, Y.; Jia, D. Fabrication of hydroxycarbonate apatite coatings with hierarchically porous structures. Acta Biomaterialia, vol. 4, issue 2, p. 334-342 (2008)

16. Marie, P.J. Strontium ranelate: a novel mode of action optimizing bone formation and resorption. Osteoporos, vol.16, issue 1, p. 7-10 (2005)

17. Ammann, P. Strontium ranelate: a novel mode of action leading to renewed bone quality. Osteoporos, vol. 16, issue 1, p. 11-15 (2005)

18. Xue, W. [et al.] Preparation and cell-materials interactions of plasma sprayed strontium-containing hydroxyapatite coating. Surface \& Coatings Technology, vol.201, issue 8, p. 4685-4693 (2007) 
19. Capuccini, C. [et al.] Strontium-substituted hydroxyapatite coatings synthesized by pulsed-laser deposition: In vitro osteoblast and osteoclast response. Acta Biomaterialia, vol.4, issue 6, p. 46854693 (2007)

20. Chen, D.M.; Fu, Y.F. Evaluation on the mechanical properties of the solid solution of strontium substituted hydroxyapatite. Chinese Journal of Stomach Materials Apparatus, vol. 19, p. 178-183 (2001)

21. Carlisle, E.M. Silicon: a possible factor in bone calcification. Science, vol 167, p. 279-280 (1970)

22. Li [et al.] Process of formation of bone-like apatite layer on silica gel. Journal of Materials Science. Materials in medicine, vol. 4, p. 127-131 (1993)

23. Thian, E.S. [et al.] Silicon-substituted hydroxyapatite: the next generation of bioactive coatings. Materials Science and Engineering: C, vol. 27, issue 2, p. 251-256 (2007)

24. Xiao, F.J. [et al.] Silicon-substituted hydroxyapatite composite coating by using vacuum-plasma spraying and its interaction with human serum albumin. Journal of Materials Science: Materials in Medicine, vol. 20, issue 8, p. 1653-1658 (2009)

25. Xiao, F.J. [et al.] Electrophoretic deposition of titanium/silicon-substituted hydroxyapatite composite coating and its interaction with bovine serum albumin. Transactions of Nonferrous Metals Society of China, vol. 19, issue 1, p. 125-130 (2009)

26. Stojanovic, D. [et al.] Bioactive glass-apatite composite coating for titanium implant synthesized by electrophoretic deposition. Journal of the European Ceramic Society, vol. 27, issue 2-3, p. 1595-1599 (2007)

27. Bharati, S. [et al.] Studies on a novel bioactive glass and composite coating with hydroxyapatite on titanium based alloys: effect of $\gamma$-sterilization on coating. Journal of the European Ceramic Society, vol. 29, issue 12, p. 2527-2535 (2009)

28. Xie, X.-H. [et al.] Enhanced osteointegration of orthopaedic implant gradient coating composed of bioactive glass and nanohydroxyapatite. Journal of Materials Science: Materials in Medicine, vol. 21, p. 2165-2173 (2010)

29. Morks, M.F. Fabrication and characterization of plasma-sprayed $\mathrm{HA} / \mathrm{SiO} 2$ coatings for biomedical application. Journal of the Mechanical Behavior of Biomedical Materials, vol. 1, issue 1, p.105-111 (2008)

30. Morks, M.F.; Fahim, N.F.; Kobayashi, A. Structure, mechanical performance and electrochemical characterization of plasma sprayed $\mathrm{SiO} 2 /$ Ti-reinforced hydroxyapatite biomedical coatings. Applied Surface Science, vol. 255, issue 5, p.3426-3433 (2008)

31. Jenkins, G.M.; De Carvalho Francisco, X. Biomedical applications of carbon fiber reinforced carbon in implanted prostheses. Carbon, vol. 15, issue 1, p.33-37 (1977)

32. Christel, P. [et al.] Development of a carbon-carbon hip prosthesis. Journal of Biomedical Materials Research, vol. 21, issue 2, p. 191-218 (1987)

33. Chen, Y. [et al.] Laser-surface-alloyed carbon nanotubes reinforced hydroxyapatite composite coatings. Applied Physics Letters, vol. 86, issue 25 , p. $251905-251905.03$ (2005)

34. Balani, K. [et al.] Plasma-sprayed carbon nanotube reinforced hydroxyapatite coatings and their interaction with human osteoblasts in vitro. Biomaterials, vol. 28, issue 4, p. 618-624 (2007)

35. Balani, K. [et al.] Tribological behaviour of plasma-sprayed carbon nanotube-reinforced hydroxyapatite coating in physiological solution. Acta Biomaterialia, vol. 3, issue 6, p. 944-951 (2007)

36. Kaya, C. Electrophoretic deposition of carbon nanotube-reinforced hydroxyapatite bioactive layers on Ti-6Al-4V alloys for biomedical applications. Ceramics International, vol. 34, issue 8, p. 1843-1847 (2008)

37. Hollinger, M.A. Toxicological aspects of topical silver pharmaceuticals. Critical reviews in toxicology, vol. 26, issue 2, p. 255-260 (1996)

38. Chen, W. [et al.] Antibacterial and osteogenic properties of silver-containing hydroxyapatite coatings produced using a sol gel process. Journal of Biomedical Materials Research Part A, vol. 82A, issue 4, p.899-906 (2007)

39. Noda, I. [et al.] Development of novel thermal sprayed antibacterial coating and evaluation of release properties of silver ions. Journal of Biomedical Materials Research part B: Applied Biomaterials, vol. 89B, issue 2, p. 456-465 (2008)

40. Teng, S. [et al.] Bioactive nanocomposite coatings of collagen/hydroxyapatite on titanium substrates. Journal of Materials Science: Materials in Medicine, vol. 19, issue 6, p. 2453-2461 (2008)

41. Kim, H.W. [et al.] Fibrillar assembly and stability of collagen coating on titanium for improved osteoblast responses. Journal of Biomedical Materials Research, vol. 75A, issue 3, p. 629-638 (2005)

42. Rammelt, S. [et al.] Coating of titanium implants with collagen, RGD peptide and chondroitin sulphate. Biomaterials, vol. 27, issue 32, p. 55615571 (2006)

43. Manara, S. [et al.] Electrochemically-assisted deposition of biomimetic hydroxyapatite-collagen coatings on titanium plate. Inorganica Chimica Acta, vol. 361, issue 6, p. 1634-1645 (2008)

44. Daugaard, H. [et al.] The effect on bone growth enhancement of implant coatings with hydroxyapatite and collagen deposited electrochemically and by plasma spray. Journal of Biomedical Materials Research Part A, vol. 92, issue 3, p. 913921 (2010) 
45. Rammelt, S.A. Coating of titanium implants with type-I collagen. Journal of Orthopaedic Research, vol. 22, p. 1025-1034 (2004)

46. Kikuchi, M. [et al.] Glutaraldehyde cross-linked hydroxyapatite/collagen self-organized nanocomposites. Biomaterials, vol. 25, issue 1, p.6369 (2004)

47. Sun, F.; Pang, X.; Zhitomirsky, I. Electrophoretic deposition of composite hydroxyapatitechitosan-heparin coatings. Journal of Materials Processing Technology, vol. 209, issue 3, p. 1597-1606 (2009)

48. Lin, W.C.; Tseng, C.H.; Yang, M.C. In vitro hemocompatibility evaluation of a thermoplastic polyurethane membrane with surface-immobilized water-soluble chitosan and heparin. Macromolecular Bioscience, vol. 5, issue 10, p. 10131021 (2005)

49. Negroiu, G. [et al.] Biocompatibility evaluation of a novel hydroxyapatite-polymer coating for medical implants (in vitro tests). Journal of Materials Science: Materials in Medicine, vol. 19, issue 4, p. 1537-1544 (2008)

50. Fleisch, H. Bisphosphonates: Mechanisms of action. Endocrine Reviews, vol. 19, issue 1, p. 80100 (1999)

51. Fleisch, H. Bisphosphonates: preclinical aspects and use in osteoporosis. Annals of Medicine, vol. 29, p.55-62 (1997)

52. Oliveira, A.L. [et al.] Biomimetic Ca-P coatings incorporating bisphosphonates produced on starch-based degradable biomaterials. Journal of biomedical materials research part B: Applied Biomaterials, vol. 92, issue 1, p. 55-67 (2009)

53. Jakobsen, T. [et al.] Local bisphosphonate treatment increases fixation of hydroxiapatitecoated implants inserted with bone compaction. Journal of Orthopaedic Research, vol. 27, issue 2, p. 189-194 (2009)

54. Sanpo, N.; Khor, K.A. [et al.] Antibacterial properties of cold-sprayed HA-Ag/PEEK coating. Journal of Thermal Spray Technology, vol. 18, issue 1, p.10-15 (2009)

55. Linsebigler, A.L.; Lu, G.; Yates, J.T. Photocatalysis on TiOn Surfaces: principles, mechanisms, and selected results. Chemical Reviews, vol. 95, p. $735-758$ (1995)

56. Breme, J. Titanium and titanium alloys, biomaterials of preference. Revue de métallurgie, vol. 10, p. 625-637 (1989)

57. Ochsenbein, A. [et al.] Osteoblast responses to different oxide coatings produced by the sol-gel process on titanium substrates. Acta Biomaterialia, vol. 4, issue 5, p. 1506-1517 (2008)

58. Jaworski, R. [et al.] Characterization of mechanical properties of suspension plasma sprayed $\mathrm{TiO} 2$ coatings using scratch test. Surface \& Coatings Technology, vol. 202, issue 12, p. 26442653 (2008)
59. Lamaka, S.V. [et al.] Nanoporous titania interlayer as reservoir of corrosion inhibitors for coatings with self-healing ability. Process in organic coatings, vol. 58, issues 2-3, p. 127-135 (2007)

60. Chiu, K.Y. [et al.] Characterization and corrosion studies of titania-coated NiTi prepared by sol-gel technique and steam crystallization. Applied Surface Science, vol. 253, issue 16, p. 6762-6768 (2007)

61. Shi, P. [et al.] Improvement of corrosion resistance of pure magnesium in Hanks' solution by microarc oxidation with sol-gel $\mathrm{TiO} 2$ sealing. Journal of Alloys and Compounds, vol. 469, issues 1-2, p. 286-292 (2009)

62. Uchida, M. [et al.] Structural dependence of apatite formation on titania gels in a simulated body fluid. Journal of Biomedical Materials Research, vol. 64A, issue 1, p. 164-170 (2003)

63. Chen, D. [et al.] Apatite formation on alkalinetreated dense $\mathrm{TiO} 2$ coatings deposited using the solution precursor plasma spray process. Acta Biomaterialia, vol. 4, issue 3, p. 553-559 (2008)

64. Li, P. [et al.] A role of hydrated silica, titania and alumina in forming biologically active bone-like apatite on implant. Journal of Biomedical Materials Research, vol. 28, issue 1, p. 7-15 (1994)

65. Wei, D. [et al.] Characteristic and in vitro bioactivity of a microarc-oxidized TiO2-based coating after chemical treatment. Acta Biomaterialia, vol. 3 , issue 5, p.817-827 (2007)

66. Henderson, M.A. Structural sensitivity in the dissociation of water on $\mathrm{TiO} 2$ single-crystal surfaces. Langmuir, vol. 12, issue 21, p. 5093-5098 (1996)

67. Liu, X. [et al.] UV-irradiation-induced bioactivity on $\mathrm{TiO} 2$ coatings with nanostructural surface. Acta Biomaterialia, vol. 4, issue 3, p. 544-552 (2008)

68. Brohede, U. [et al.] A novel graded bioactive high adhesion implant coating. Applied Surface Science, vol. 255, issue 17, p. 7723-7728 (2009)

69. Cavalcanti-Adam E.A. [et al.] Cell adhesion and response to synthetic nanopatterned environments by steering receptor clustering and spatial location. HFSP Journal, vol. 2, issue 5, p. 276285 (2008)

70. Von der Mark, K. [et al.] Nanoscale engineering of biomimetic surfaces : cues from the extracellular matrix. Cell and Tissue Research, vol. 339, issue 1, p. 131-153 (2010)

71. Advincula, M.C. [et al.] Surface analysis and biocorrosion properties of nanostructured surface sol-gel coatings on Ti6A14V Titanium alloy implants. Journal of biomedical materials research. Part B: Applied biomaterials, vol. 80, issue 1, p. 107-120 (2007)

72. Crawford, G.A. [et al.] Microstructure and deformation behavior of biocompatible $\mathrm{TiO} 2$ nanotubes on titanium substrate. Acta Biomaterialia, 
vol. 3, issue 3, p. 359-367 (2007)

73. Crawford, G.A.; Chawla, N. Porous hierarchical $\mathrm{TiO} 2$ nanostructures: processing and microstructure relationships. Acta Materialia, vol. 57, issue 3, p. 854-867 (2009)

74. Crawford, G.A.; Chawla, N.; Houston, J.E. Nanomechanics of biocompatible $\mathrm{TiO} 2$ nanotubes by Interfacial Force Microscopy (IFM). Journal of the mechanical behavior of biomedical materials, vol. 2, issue 6, p. 580-587 (2009)

75. Von Wilmowski, C. [et al.] In vivo evaluation of anodic TiO2 nanotubes: an experimental study in the pig. Journal of Biomedical Materials Research, Part B: applied biomaterials, vol. 89B, issue 1, p. 165-171 (2009)

76. Ma, Q. [et al.] Enhancement of the bioactivity of titanium oxide nanotubes by precalcification. Materials Letters, vol. 63, issues $17-18$, p. 30353038 (2008)

77. Huang, Z. [et al.] Effect of nanofiber-coated surfaces on the proliferation and differentiation of osteoprogenitors in vitro. Tissue Engineering: Part A, vol. 14, issue 11, p. 1853-1859 (2008)

78. Bienias, J. [et al.] The influence of $\mathrm{SiO} 2$ and $\mathrm{SiO} 2-\mathrm{TiO} 2$ intermediate coatings on bond strength of titanium and Ti6Al4V alloy to dental porcelain. Dental Materials, vol. 25, issue 9, p. 11281135 (2009)

79. Aaritalo, V. [et al.] Sol-gel derived $\mathrm{TiO} 2-\mathrm{SiO} 2$ implant coatings for direct tissue attachment. Part I: design, preparation and characterization. Journal of Materials Science. Materials in Medicine, vol. 18, issue 9, p. 1863-1873 (2007)

80. Areva, S. [et al.] Sol-gel derived $\mathrm{TiO} 2-\mathrm{SiO} 2 \mathrm{im}-$ plant coatings for direct tissue attachment. Part II: evaluation of cell response. Journal of Materials Science. Materials in Medicine, vol. 18, issue 8, p. 1633-1642 (2007)

81. Wei, D. [et al.] Formation of $\mathrm{CaTiO} 3 / \mathrm{TiO} 2 \mathrm{com}-$ posite coating on titanium alloy for biomedical applications. Journal of biomedical materials research, part B: applied biomaterials, vol. 84B, issue 2, p. 444-451 (2008)

82. Necula, B.S. [et al.] In vitro antibacterial activity of porous $\mathrm{TiO} 2-\mathrm{Ag}$ composite layers against methicillin-resistant Staphylococcus aureus. Acta Biomaterialia, vol. 5, issue 9, p. 3573-3580 (2009)

83. Yuan, S.J. [et al.] Biocorrosion behaviour of titanium oxide/butoxide-coated stainless steel. Journal of the electrochemical society, vol. 155, issue 5, p. 196-210 (2008)

84. Zhang, W. [et al.] Tribological behaviors of single and dual sol-gel ceramic film on Ti-6Al-4V. Ceramics International, vol. 35, issue 4, p. 1513 1520 (2009)

85. Wen, C.E. [et al.] Hydroxyapatite/titania sol-gel coatings on titanium-zirconium alloy for biomedical applications. Acta Biomaterialia, vol. 3, is- sue 3, p. 403-410 (2007)

86. He, G. [et al.] Surface modification of titanium by nano-TiO2/HA bioceramic coating. Applied Surface Science, vol. 255, issue 2, p. 442-445 (2008)

87. Tomaszek, R. [et al.] Microstructure of suspension plasma sprayed multilayer coatings of hydroxyapatite and titanium oxide. Surface and Coatings Technology, vol. 201, issues 16-17, p. 7432-7440 (2007)

88. Lee, S.H.; Kim, H.E.; Kim, H.W. Nano-sized hydroxyapatite coatings on $\mathrm{Ti}$ substrate with $\mathrm{TiO} 2$ buffer layer by E-beam deposition. Journal of the American Ceramic Society, vol. 90, issue 1, p. 50-56 (2007)

89. Wang, Y.-Q. [et al.] HA coating on titanium with nanotubular anodized $\mathrm{TiO} 2$ intermediate layer via electrochemical deposition. Transactions of Nonferrous Metals Society of China, vol. 18, issue 3, p. 631-635 (2008)

90. Albayrak, O.; El-Atwani, O.; Altintas, S. Hydroxyapatite coating on titanium substrate by electrophoretic deposition method: effects of titanium dioxide inner laye ron adhesión strength and hydroxyapatite decomposition. Surface and Coatings Technology, vol. 202, issue 11, p. 24822487 (2008)

91. Zhu, L. [et al.] Biomimetic coating of compound titania and hydroxyapatite on titanium. Journal of Biomedical Materials Research, vol. 83A, issue 4, p. 1165-1175 (2007)

92. Sun, T.; Wang, M. Low-temperature biomimetic formation of apatite/TiO2 composite coatings on $\mathrm{Ti}$ and NiTi shape memory alloy and their characterization. Applied Surface Science, vol. 255, issue 2, p. 396-400 (2008)

93. Im, K.-H. [et al.] Improvement of bonding strength to titanium surface by sol-gel derived hybrid coating of hydroxyapatite and titania by sol-gel process. Surface and Coatings Technology, vol. 202, issue 4-7, p. 1135-1138 (2007)

94. Han, J.Y.; Yu, Z.T.; Zhou, L. Hydroxyapatite/ titania composite bioactivity coating processed by sol-gel method. Applied Surface Science, vol. 255, issue 2, p. 455-458 (2008)

95. Joseph Nathanael, A.; Mangalaraj, D.; Ponpandian, N. Controlled growth and investigations on the morphology and mechanical properties of hydroxyapatite/titania nanocomposite thin films. Composites Science and Technology, vol. 70, p. 1645-1651 (2010)

96. Gaona, M.; Lima, R.S.; Marple, B.R. Nanostructured titania/hydroxyapatite composite coatings deposited by high-velocity oxy-fuel (HVOF) spraying. Materials Science and Engineering A, vol. 458, issues 1-2, p. 141-149 (2007)

97. Lima, R.S. [et al.] HVOF-Sprayed nano TiO2HA coatings exhibiting enhanced biocompatibili- 
ty. Journal of Thermal Spray Technology, vol. 19, issues 1-2, p. 336-342 (2010)

98. Boyd, A.R. [et al.] Characterisation of calcium phosphate/titanium dioxide hybrid coatings. Journal of Materials Science: Materials in Medicine, vol. 19, issue 2, p. 485-498 (2008)

99. Boyd, A.R. [et al.] Sputter deposition of calcium phosphate/titanium dioxide hybrid thin films. Materials Science and Engineering C, vol. 28, issue 2, p. 228-236 (2008)

100. Kim, D.-Y. [et al.] Formation of hydroxyapatite within porous $\mathrm{TiO} 2$ layer by micro-arc oxidation coupled with electrophoretic deposition. Acta Biomaterialia, vol. 5, issue 6, p. 2196-2205 (2009)

101. Cheng, K. [et al.] Sol-gel preparation and in vitro test of fluorapatite/hydroxyapatite films. Journal of Biomedical Materials Research, part B: applied biomaterials, vol. 69B, issue 1, p. 33-37 (2003)

102. Kim, H.-W.; Kim, H.-E.; Knowles, J.C. Fluorhydroxyapatite sol-gel coating on titanium substrate for hard tissue implants. Biomaterials, vol. 25 , issue 17, p. 3351-3358 (2004)

103. Wang, J. [et al.] Fluoridated hydroxyapatite/titanium dioxide nanocomposite coating fabricated by a modified electrochemical deposition. Journal of Materials Science: Materials in Medicine, vol. 20, issue 5, p. 1047-1055 (2009)

104. Mo, A. [et al.] Preparation and antibacterial effect of silver-hydroxyapatite/titania nanocomposite thin film on titanium. Applied Surface Science, vol. 255, issue 2, p. 435-438 (2008) 